\title{
Absorption-line-locked fiber laser for gas-sensing applications
}

\author{
R. Romero, ${ }^{*}$ F. M. Araújo, and L. A. Ferreira \\ INESC Porto, Rua do Campo Alegre 687, 4169-007 Porto, Portugal \\ ${ }^{*}$ Corresponding author: rromero@multiwavephotonics.com \\ Received 24 July 2007; accepted 28 August 2007; \\ posted 28 September 2007 (Doc. ID 85554); published 8 November 2007
}

\begin{abstract}
An absorption-line-locked fiber laser based on the selection and conversion of a gas absorption line into a highly stable intracavity transmission narrowband filter is demonstrated. The laser has attractive properties for gas-sensing applications due to its frequency stability and multiwavelength selection capability. (C) 2007 Optical Society of America

OCIS code: 060.2340 .
\end{abstract}

\section{Introduction}

The development of laser sources with highly stabilized emission wavelength to be used in gas-sensing applications is of major interest for applications such as breath diagnostics, process and air quality, or atmospheric pollution monitoring $[1,2]$. In general, the high power emitted by laser sources, as well as their narrow linewidth and reliability, make them the ideal sources for those applications. However, environmental spectral sensitivity demands the implementation of active wavelength-locking schemes to ensure the stability necessary to provide proper species selectivity.

The simplest frequency stabilization technique consists in isolating the laser cavity from environment influence, the most critical being temperature-induced fluctuations. Other techniques of frequency stabilization are based on using a saturated absorption-line component [3], another laser [4], or a cavity [5] as frequency references. Alternative methods such as phase-locking schemes [6], spectral hole burning [7], or Zeeman effects [8] have also been proposed to stabilize the laser output at a certain frequency. Pursuing the same objective, Kim et al. proposed the introduction of a Mach-Zehnder interferometer in the cavity of the laser [9].

Michelson interferometer configurations have been widely used in sensing applications [10], interroga-

0003-6935/07/327900-06\$15.00/0

(C) 2007 Optical Society of America tion systems [11], or telecommunications devices [12]. This interferometric configuration can also be used for the fabrication of optical filters. Bilodeau et al. proposed a narrowband all-fiber bandpass transmission filter by interfering light reflected from identical gratings placed in each arm [13].

In this paper an absorption-line-locked fiber laser based on the use of a gas absorption line combined with a Michelson configuration is proposed. The device converts a given gas absorption line into a highly stabilized intracavity filter to define the emission wavelength of the laser source. The inherent low temperature dependence of gas absorption lines allows the implementation of low power consumption and compact passive systems while ensuring the frequency stability required for gas-sensing applications. By taking advantage of inherent low temperature dependence of the gas absorption line, the proposed concept should allow frequency stability in the range of 1 part in $10^{8}$.

\section{Wavelength Selection Concept}

The principle behind the wavelength stabilization is to select and convert a given gas absorption line into a highly stable transmission filter. To achieve such a feature, a fiber-coupled gas cell containing the relevant mixture is inserted into one arm of a balanced and stabilized Michelson interferometer as shown in Fig. 1. In general, when light from a broadband source is launched into an exactly balanced interferometer, then one output port sees totally con- 


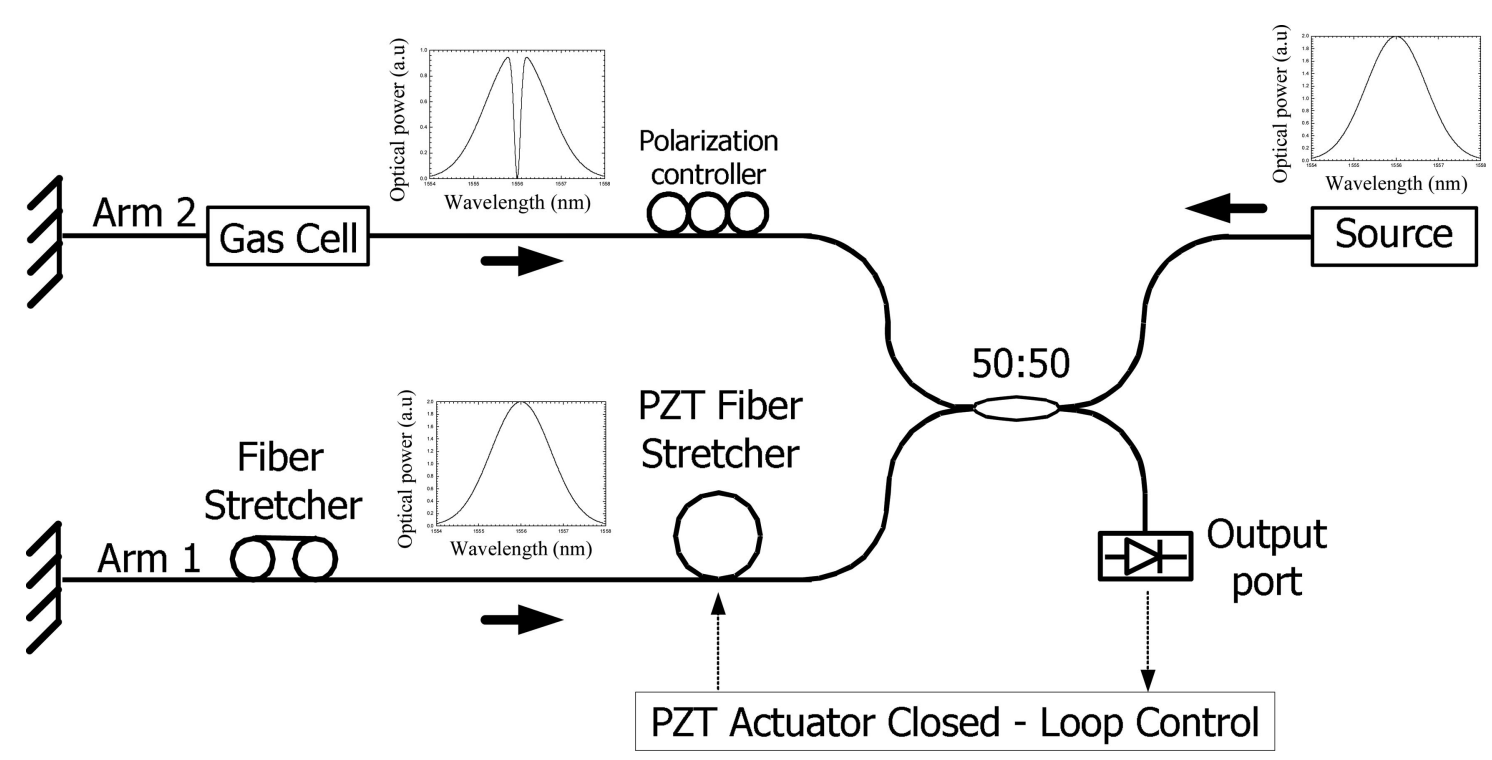

Fig. 1. Absorption-line-locked fiber filter configuration.

structive interference, while the other sees totally destructive interference regardless of wavelength. The insertion of the gas cell in one arm of the interferometer leads to the absorption of part of the light, giving rise to spectrally different signals on both arms of the interferometer. The absence of selectively absorbed light in the arm that contains the gas cell prevents interference in such a spectral band, so corresponding light coming from the other arm can never be canceled. In this way, the gas absorption band is effectively converted into a transmission band. It must be emphasized that this conversion is effective even if the interferometer is not exactly balanced, since it is only necessary to ensure that the interference fringe spectral period is large when compared with the absorption bandwidth. The selection of the output port where the absorption feature is effectively converted into a transmission narrowband line is set by ensuring destructive interference at that output. This can be achieved by acting differentially on the interferometer arms so that the interfering signals are in-phase or out-of-phase.

Important factors in the design of this line-locked filter are the light power balance on both arms and the isolation level of the absorption line that is introduced in one of the arms. Simulations were done in order to understand the spectral response of the filter addressing different design conditions. Figure 2 shows the most relevant filter design conditions, evidencing the spectral power density in each arm prior to the interference at the coupler (left) and the spectral response of the Michelson interferometer for inphase and out-of-phase interference (right). Case (a) simulates the optimum design condition in which both arms have exactly the same power balance and the absorption line has $100 \%$ isolation level. When in-phase interference occurs, the corresponding output replicates the spectral power density of the light source besides the Lamb dip associated with the ab- sorption line (Fig. 2(a): in-phase interference). In the conditions of out-of-phase interference, the output is limited to the light that does not interfere due to the absorption line (Fig. 2(a): out-of-phase interference). In a practical implementation it is necessary to evaluate the effect of power unbalance both due to the coupler spectral response and the insertion losses in the gas cell. Moreover, each line is characterized by a particular absorption cross section that results in an effective round-trip absorption ratio that departures from $100 \%$ extinction. Power unbalance results in a pedestal in the spectral response of the filter for the out-of-phase interference condition and a peak height ratio reduction for the in-phase condition. The unabsorbed light produces a power gap between the in-phase and out-of-phase responses. The conjunction of both of these effects is shown in Fig. 2(b), where both arms have different power balance and the absorption line has an isolation level of $50 \%$. As can be seen, the intensity power decreases and a power gap between in-phase and out-of-phase interference cases appears.

\section{Experimental Filter Response}

The setup shown in Fig. 1 was used for the implementation of the proposed absorption-line-locked fiber transmission filter concept. An erbium-doped broadband optical source (BBOS) and an acetylene gas cell were used for demonstration purposes. The spectral response of the acetylene gas cell is shown in Fig. 3. It can be noticed that the spectrum has several absorption lines with different isolation levels at different wavelengths. This feature enables a flexible selection of one of multiple absorption lines to define a specific wavelength transmission filter through the use of wavelength-selective mirrors in the arms of the Michelson interferometer, e.g., fiber Bragg gratings (FBGs) or thin film mirrors. In the present demonstration, silver-based reflection coatings were 

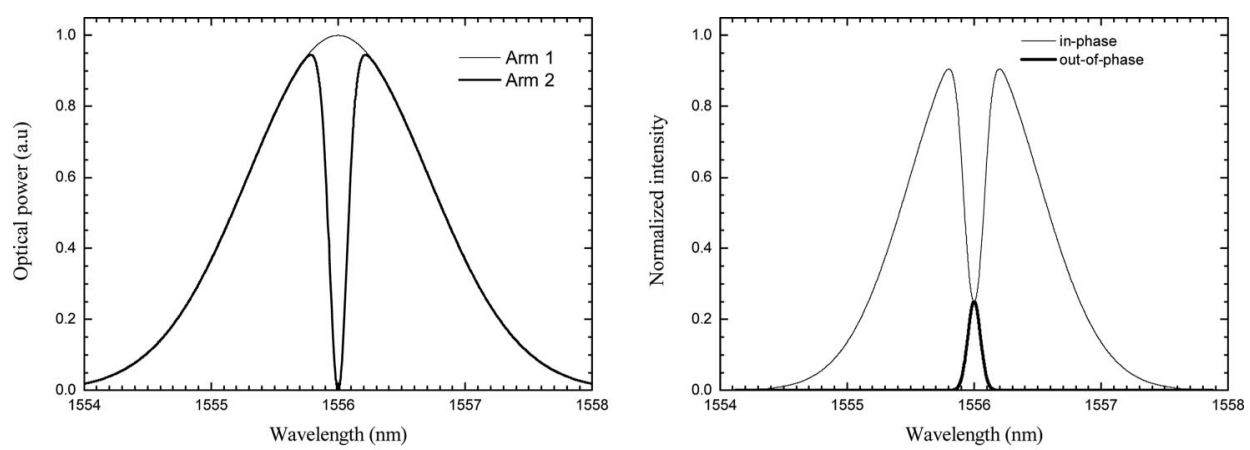

(a)
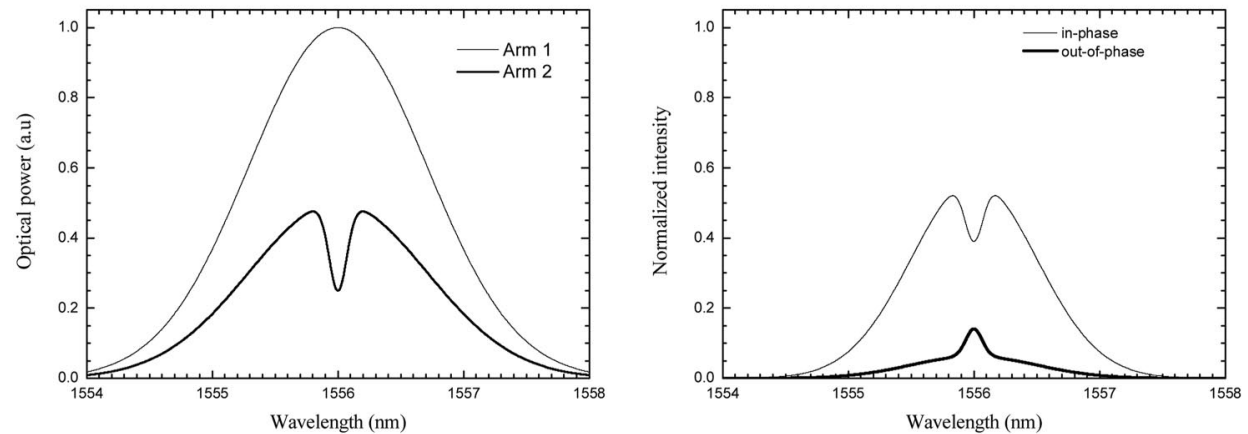

(b)

Fig. 2. Simulation of the absorption-line-locked fiber filter spectral response for (a) optimum design and (b) practical conditions. (left) Spectral power density prior to interference at the coupler. (right) Interference signals for in-phase and out-of-phase cases.

deposited at both fiber ends with approximately $85 \%$ reflectivity, being a FBG, with a center wavelength at $1535.3 \mathrm{~nm}$, used as the laser wavelength-selective output coupler. The center wavelength of this Bragg grating was chosen in order to overlap one of the absorption lines of the acetylene gas cell. To avoid thermal-induced Bragg wavelength drift, proper FBG protection was implemented. Practical system implementation can take advantage of commercially available passive athermal FBG packaging.

Two fiber stretchers were used in the setup shown in Fig. 1, one of them based on a manual translation stage and the other consisting of a piezoelectric transducer (PZT) controlled by a high voltage power supply. This assembly allows the effective control of the path imbalance between both arms of the interferometer not only to achieve proper balancing but also to adjust the interferometric phase. A polarization controller was introduced in one of the arms in order to optimize fringe visibility. In a practical system, this can be avoided by inserting Faraday rotator mirrors in the arms of the Michelson interferometer [14].

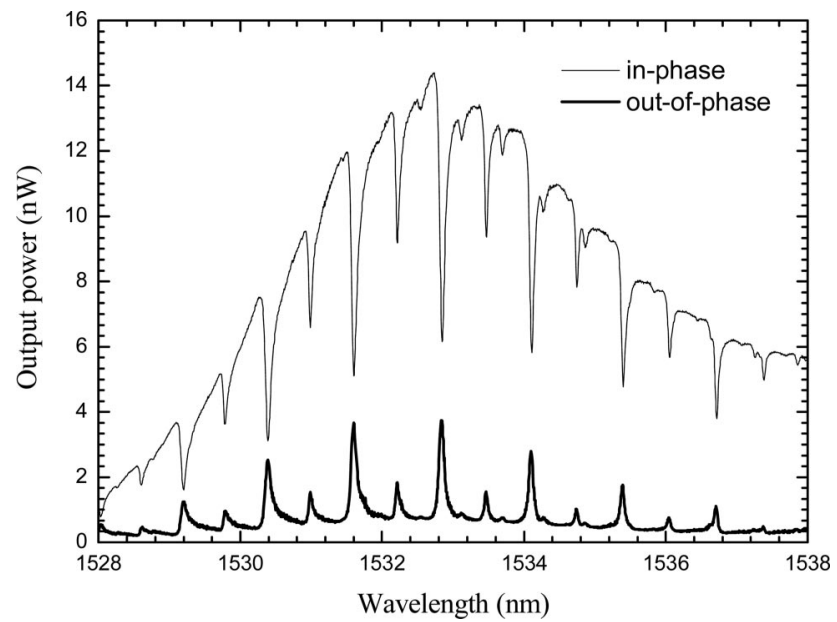

Fig. 4. In-phase and out-of-phase output of the absorption-linelocked filter measured with an OSA. 


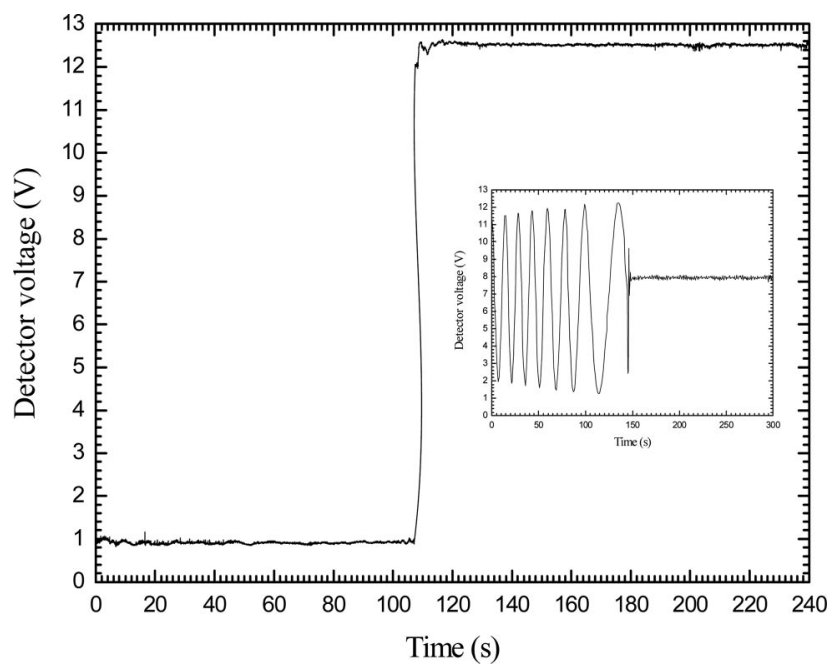

Fig. 5. Detector voltage output for in-phase and out-of-phase locking conditions. (inset) Servo off and on.

Figure 4 shows the spectral response of the filter for in-phase and out-of-phase interference conditions measured with an optical spectrum analyzer (OSA) with $15 \mathrm{pm}$ resolution at the output port. Noticeable is a pedestal at destructive interference conditions caused by the unbalanced power in both arms of the interferometer. This problem arises from fiber coupling loss in the gas cell, a coupler spectral response, and different splicing losses when connecting all the elements of the filter. On the other hand, an expected power gap between the in-phase and out-of-phase interferences is also observed. This power gap is because the acetylene gas cell has a maximum of $48 \%$ of isolation level (see Fig. 3). This behavior was already predicted in Fig. 2(b).

Path fluctuations between both arms of the interferometer arise mainly from environmentally induced temperature drift in the arms of the interferometer. A digital servo system (Fig. 1) was introduced in the system to compensate this effect: The optical power at the output of the interferometer is measured with a photodetector, digitalized, and acquired through a

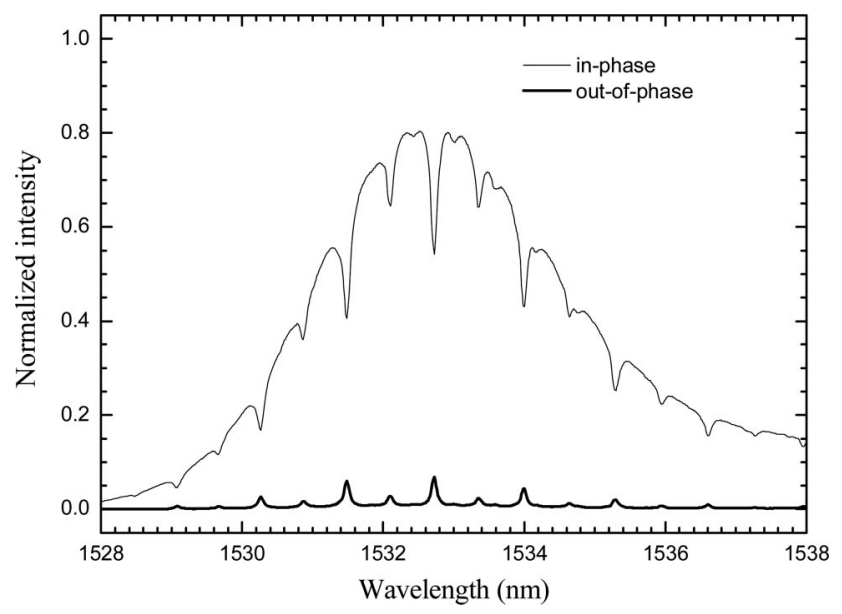

Fig. 6. Theoretical in-phase and out-of-phase interference.

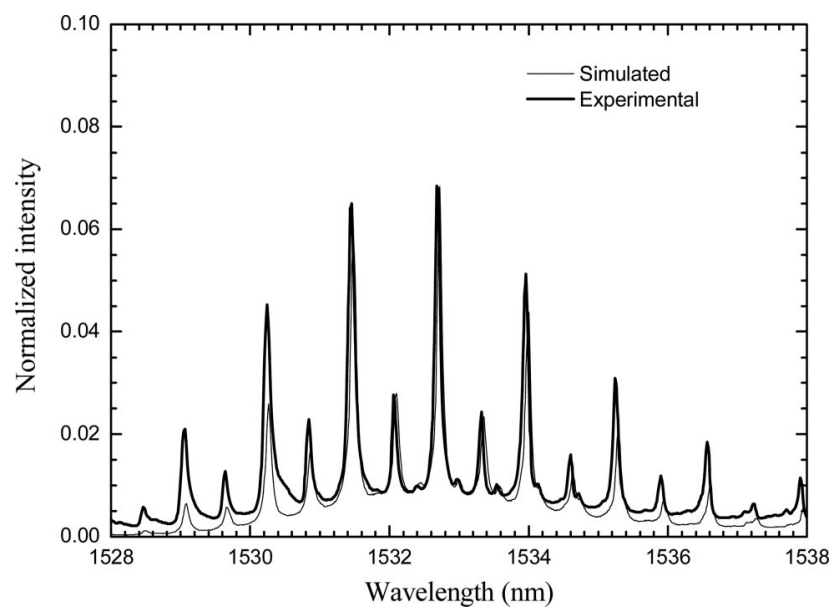

Fig. 7. Comparison between theoretical and experimental results for destructive interference.

standard data acquisition board; any deviation from the locking value is then computed and integrated at software level to define an error signal that is applied to the PZT fiber stretcher to control the path imbalance. It must be emphasized that the frequencylocking mechanism is completely passive, this servo being used only for power stabilization purposes.

Figure 5 shows the locking voltage signal for destructive and constructive interference conditions. These locking conditions are difficult to achieve with the simple homodyne servo system implemented because they correspond to the edges of the interferometer transfer function. In the inset of Fig. 5, it is possible to observe the effect on the detector signal of a transition from a fluctuating situation to a locked condition achieved when the servo system is activated. The visibility of the interferometer was experimentally determined to be $\sim 90 \%$.

The power unbalance between the two arms of the interferometer was estimated experimentally to be $\sim 80 \%$ by consecutively canceling light in each arm. Using this value, the spectral responses of the gas cell and the broadband source emulated from experimental data, it is possible to calculate the theoretical spectral response at the output of the interferometer. The computed interference signal is shown in Fig. 6 for both for in-phase and out-of-phase interference conditions.

Figure 7 compares this theoretical result with experimental data. From this figure, it is possible to conclude that the experimental results are in good agreement with the theoretical model predicted for the line-locked filter. In forthcoming sections this filter will be used as the key element in the configuration of a frequency-stabilized fiber laser.

\section{Absorption-Line-Locked Fiber Laser Configuration}

Figure 8 shows the proposed absorption-line-locked fiber laser configuration. The line cavity is formed by the absorption fiber filter presented in previous sections, $35 \mathrm{~m}$ of erbium-doped fiber (EDF), a wave- 


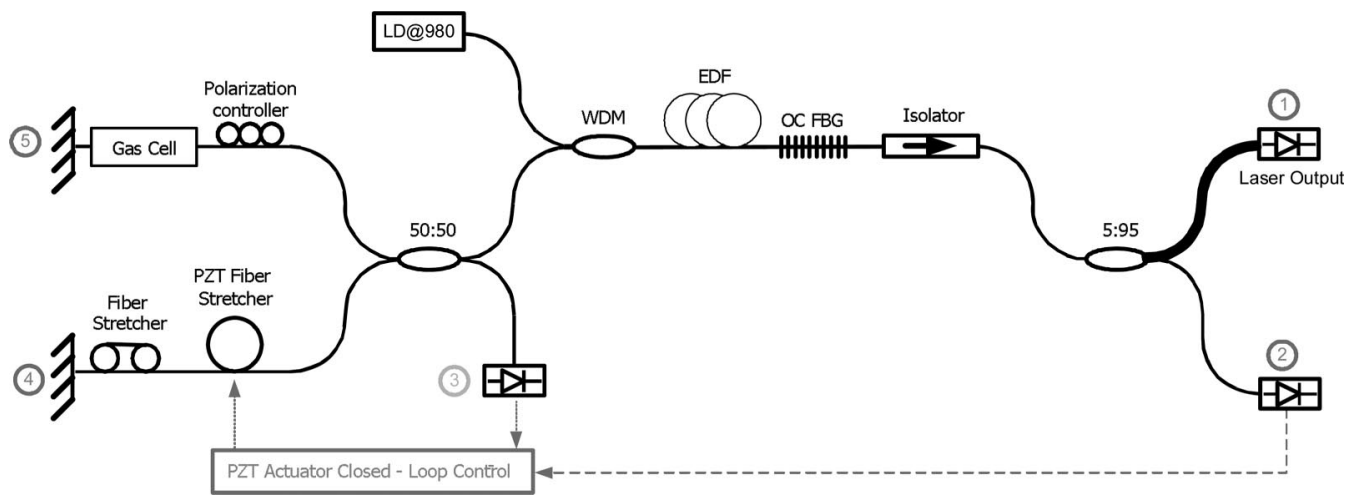

Fig. 8. Cavity configuration proposed for the absorption-line-locked fiber laser.

length division multiplexer for pumping light into the EDF, a FBG with $80 \%$ reflectivity, and an isolator to prevent backward reflections.

In a first stage, both arms of the interferometer side of the in-line cavity are balanced to the same length with the help of the manual translation stage and the loop control that uses the power coming out from output 3 (see Fig. 8). This procedure is the same as the one explained before, where any deviation from the locked voltage is taken as an error signal, which is applied to the PZT fiber stretcher.

To assure an appropriate behavior of the locked laser, the out-of-phase interference signal from the interferometer should travel through the in-line cavity. By closing the loop with the voltage output from port 2 (see Fig. 8), the servo control signal can be set for maximum or minimum output voltages (Fig. 9). In this case, the maximum output voltage of the loop corresponds to introducing the in-phase interference in the cavity, which is not desirable for the proper working regime of the laser. On the other hand, the minimum output voltage corresponds to introducing the out-of-phase interference into the in-line cavity, simultaneously allowing the selection of the emitting frequency and the power output stabilization of the in-line locked fiber laser. As can be seen, the mini-

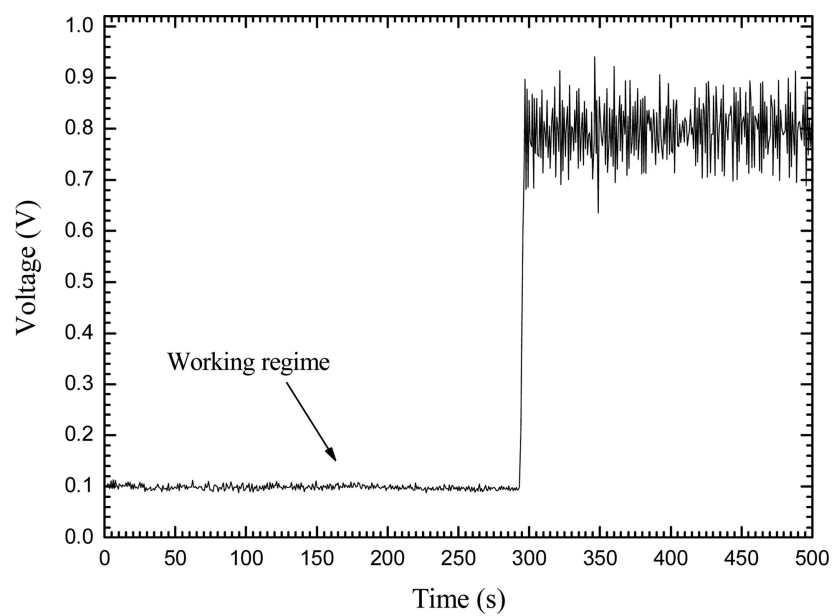

Fig. 9. Detector voltage output for locking conditions in output 2 (see Fig. 8). mum voltage-locked position is much more stable than the maximum voltage-locked position In fact, this last regime, which corresponds to introducing the in-phase interference in the in-line cavity, is very unstable and therefore not desirable for a frequencystabilized laser.

The different voltage scales in Fig. 5 and Fig. 9 are due to different photodetector amplifications of the optical output. When the output voltage of port 2 is used to close the loop, a small amplification is used because of the laser's higher output power, and therefore the voltage scale in the graphic is smaller.

It should also be mentioned that when the signal obtained from output port 3 is used in the loop, from the maximum and minimum lock voltage values it is possible to determine the interferometer visibility.

The frequency stability of the locked laser depends only on the stability of the absorption spectrum of the gas cell. In general, gas absorption lines are used as primary wavelength references, as they present very low intrinsic drift. In this particular case, the acetylene gas cell used is insensitive to atmospheric pressure or humidity variations and has a temperature dependence lower than $0.01 \mathrm{pm} /{ }^{\circ} \mathrm{C}$ [15]. Therefore the proposed laser configuration should be capable of achieving frequency stability of the order of $1 \mathrm{MHz}$

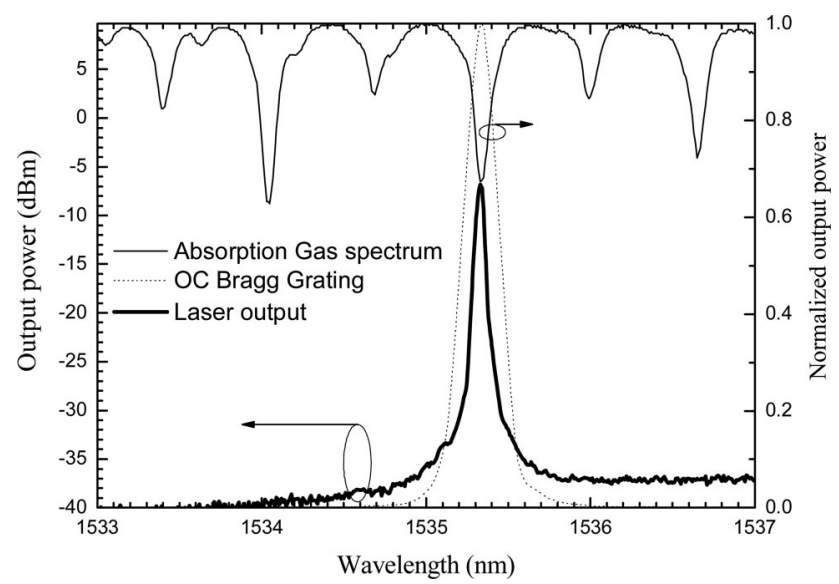

Fig. 10. Output fiber laser at $1535.3 \mathrm{~nm}$ (solid curve), normalized absorption spectrum of the acetylene gas cell (line) and reflection spectrum of the Bragg grating (dot curve). 


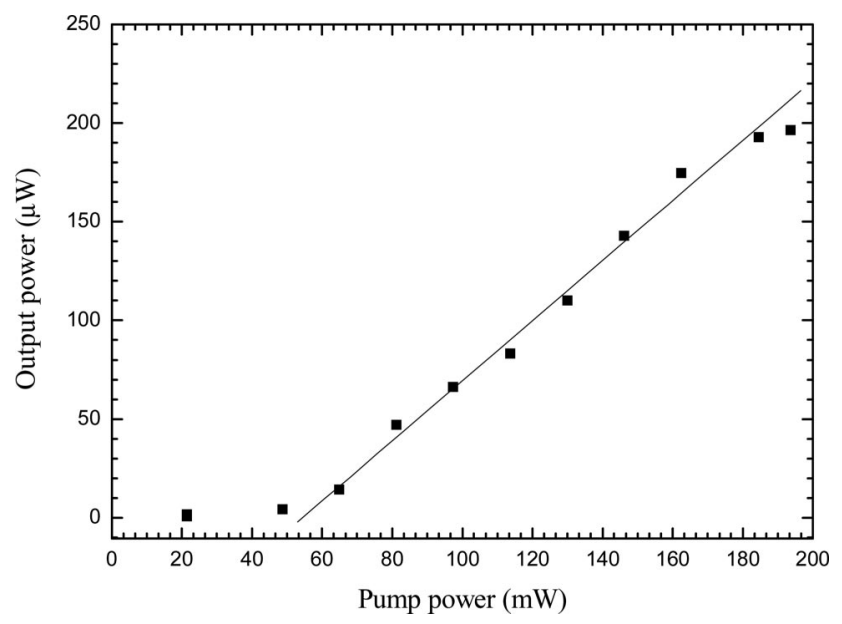

Fig. 11. Output power of the fiber laser versus input power of the pump laser diode.

( 1 part in $10^{8}$ ). Further measurements should be accomplished in order to confirm this statement.

Figure 10 shows the output power at the locked frequency for the maximum pump power of the pump laser. It also shows the normalized absorption spectra of the acetylene gas cell, and it can be observed that the output linewidth is locked at the chosen absorption line of the gas cell.

Figure 11 shows the frequency-stabilized laser output power versus the pump power. Since the cavity design was not optimized, the obtained efficiency was low $(0.1 \%)$, but for each power value, the frequency and power remained constant over time.

The frequency and power stability behavior obtained can be applied in many sensing applications, e.g., the monitoring of different gases in the atmosphere, as the absorption gas cell in the locked laser can be changed within necessities.

\section{Conclusion}

In this work a passive absorption-line-locked fiber transmission filter was demonstrated. The proposed concept allows the selection and conversion of a given gas absorption line into a highly stable transmission filter. The demonstrated configuration based on an acetylene gas cell allowed the implementation of a multiwavelength transmission filter.

This transmission filter was exploited as an intracavity element to define the emission wavelength of a fiber laser source. This source could be used for gas sensing applications. In this way a perfect match between the laser emission wavelength and the gas species is ensured without the need of any active wavelength-locking schemes. A frequency stability of 1 part in $10^{8}$ was achieved.

The Fundação para a Ciência e Tecnologia (FCT) is acknowledged for providing support to this work through the postdoctoral grant SFRH/B PD/16505/ 2004 and the research project OPTOPACK (POSC/ EEA-ESE/61449/2004).

\section{References}

1. G. Stewart, G. Whitenett, P. Shields, J. Marshall, and B. Culshaw, "Industrial and highway sensors technology," in Design of Fiber Laser and Sensor Systems for Gas Spectroscopy in the Near-IR, Proc. SPIE 5272, 172-180 (2003).

2. K. Chan, H. Ito, and H. Inaba, "An optical-fiber-based gas sensor for remote absorption measurement of low-level CH4 gas in the near-infrared region," J. Lightwave Technol. 2, 234237 (1984).

3. S. L. Gilber, "Frequency stabilization of a tunable erbiumdoped fiber laser," Opt. Lett. 16, 150-152 (1991).

4. J. Rauschenberger, T. M. Fortier, D. J. Jones, J. Ye, and S. T. Cundiff, "Control of the frequency comb from a modelocked erbium-doped fiber laser,” Opt. Express 10, 1404-1410 (2002)

5. S. Seel, R. Storz, G. Ruoso, J. Mlynek, and S. Schiller, "Cryogenic optical resonators: a new tool for laser frequency stabilization at the $1 \mathrm{~Hz}$ level," Phys. Rev. Lett. 78, 4741-4744 (1997).

6. B. R. Washburn, S. A. Diddams, N. R. Newbury, J. W. Nicholson, M. F. Yan, and C. G. Jørgensen, "Phase-locked, erbiumfiber-laser-based frequency comb in the near infrared," Opt. Lett. 29, 250-252 (2004).

7. N. M. Strickland, P. B. Sellin, Y. Sun, J. L. Carlsten, and R. L. Cone, "Laser frequency stabilization using regenerative spectral hole burning," Phys. Rev. B 62, 1473-1476 (2000).

8. V. V. Yashchuk, D. Budker, and J. R. Davis, "Laser frequency stabilization using linear magneto-optics," Rev. Sci. Instrum. 71, 341-346 (2000).

9. S. K. Kim, G. Stewart, W. Johnstone, and B. Culshaw, "Modehop-free single-longitudinal-mode erbium-doped fiber laser frequency scanned with a fiber ring resonator," Appl. Opt. 38, 5154-5157 (1999).

10. R. Kashyap and B. Nayar, "An all single-mode fiber Michelson interferometer sensor," J. Lightwave Technol. 1, 619-624 (1983).

11. J. L. Santos, F. Farahi, T. Newson, A. P. Leite, and D. A. Jackson, "Frequency multiplexing of remote all-fiber Michelson interferometers with lead insensitivity," J. Lightwave Technol. 10, 853-863 (1992).

12. C. Watts and N. Furstenau, "Multistable fiber-optic Michelson interferometer with electrooptic feedback exhibiting 95 stable states," J. Quantum Electron. 25, 3-5 (1989).

13. F. Bilodeau, K. O. Hill, B. Malo, D. C. Johnson, and J. Albert, "High-return-loss narrowband all-fiber bandpass transmission filter," Photonics Technol. Lett. 6, 80-82 (1994).

14. A. D. Kersey, M. J. Marrone, and M. A. Davis, "Polarization insensitive fiber optic Michelson interferometer," Electron. Lett. 27, 518-519 (1991).

15. "C-band gas cell acetylene cells: C2H2-M," wavelength references data sheet file: Acetylene.pdf. 